EPJ Web of Conferences 116, 05006 (2016)

DOI: $10.1051 /$ epjconf/201611605006

(C) Owned by the authors, published by EDP Sciences, 2016

\title{
Characterization of the electro-optical transceivers in the KM3NeT optical network
}

\author{
S. Pulvirenti ${ }^{1, \mathrm{a}}$, F. Ameli ${ }^{2}$, A. D’Amico ${ }^{3}$, G. Kieft ${ }^{3}$, and J.-W. Schmelling ${ }^{3}$ for KM3NeT Collaboration \\ ${ }^{1}$ INFN Laboratori Nazionali del Sud, via S. Sofia 62, 95123 Catania, Italy \\ ${ }^{2}$ INFN Sezione Roma, P.le A. Moro 2, 00185 Roma, Italy \\ ${ }^{3}$ Nikhef, Science Park 1051098 XG, Amsterdam, The Netherlands
}

\begin{abstract}
KM3NeT is a future research infrastructure hosting a network of neutrino telescopes in the abyss of the Mediterranean Sea. The whole data transport over an optical network is based on the Dense Wavelength Division Multiplexing technique with optical channels spaced $50 \mathrm{GHz}$ apart and a bit rate of $1.25 \mathrm{Gbps}$. Over the telescope lifetime, precise temperature control of the laser is required to maintain stability of the central frequency, complying with the recommendations of the International Telecommunication Union. We will report on the characterisation of the tuneable features of the electro-optical transceivers required to compensate for the expected wavelength drift due to aging factors. Preliminary results will be showed.
\end{abstract}

\section{Introduction}

KM3NeT is a large second-generation detector in the abyss of the Mediterranean Sea. The first phase of the project (Phase-1) is funded mainly by Italian, Dutch and French contributions and foresees the construction and the deployment of 31 Detection Units (DU), 24 at the KM3NeT-It site (off-shore Portopalo di Capo Passero) and the remaining at the KM3NeT-Fr site (off-shore Toulon). The KM3NeTIt site will host also a set of 8 DUs constructed according to a previous design (NEMO tower [1]), which will be operated independently [2]. The data transfer over an optical network is based on the Dense Wavelength Division Multiplexing (DWDM) technique for both sites. The communication between on and off shore electronics is established over optical fibre using Small Form Factor Pluggable (SFP) transceiver. The one used in the DWDM network has optical channels spaced $50 \mathrm{GHz}$ apart, a bit rate of $1.25 \mathrm{Gbps}$ and up to $100 \mathrm{~km}$ span length. Over the telescope lifetime, precise temperature control of the laser is required to maintain stability of the central frequency, complying with the ITU-T G.694.1 [3] recommendations of the International Telecommunication Union.

\footnotetext{
a e-mail: spulvirenti@lns.infn.it
}

This is an Open Access article distributed under the terms of the Creative Commons Attribution License 4.0, which permits unrestricted use, distribution, and reproduction in any medium, provided the original work is properly cited. 


\begin{tabular}{|c|c|c|c|c|c|}
\hline & \multicolumn{5}{|l|}{ Requirements: } \\
\hline & & $\min$ & typ & $\max$ & \\
\hline & Wavelength range & 1528 & & 1564 & $\mathrm{~nm}$ \\
\hline & Extinction ratio & 8 & & & dB \\
\hline & Output power & 0 & & $\mathrm{~s}$ & $d B m$ \\
\hline OE $\underset{*}{*}$ & Deviation From Central Frequency@EOL ${ }^{1}$ & -6 & & +6 & $\mathrm{GHz}$ \\
\hline SOLUTIONS & Bitrate transmitter ${ }^{2}$ & 1.25 & & & Gbps \\
\hline & Link budget & 24 & & & $\mathrm{~dB}$ \\
\hline & Lifetime & 15 & & & year \\
\hline & ITU channel spacing & & & 50 & $\mathrm{GH} z$ \\
\hline & Power consumption & & 1 & 1.2 & Watt \\
\hline
\end{tabular}

Figure 1. Left: Picture of the OESolutions SFP transceiver used in the KM3NeT project. Right: main SFP requirements for the project optical network.

\section{The Electro-optical transceivers in $\mathrm{KM} 3 \mathrm{NeT}$}

The SFP transceivers used in KM3NeT optical network [4] are from OESolutions (see picture in Fig. 1) whose distributor for the project is Tallgrass [5]. Main KM3NeT SFP requirements are reported in Fig. 1.

OESolutions provides a digital diagnostic monitoring interface which allows real time access to the device operating parameters such as the transmitted and received optical power, the laser temperature, the supply voltage and the Thermo Electric Cooler (TEC) controller current.

\section{Wavelength Tuning and fist test at Nikhef}

The wavelength tuning feature of a SFP is a special aspect which is not required by the DWDM SFP Multiple Source Agreement (MSA) standards. The principle of the wavelength tuning is based on changing the temperature of the Transmission Optical SubAssembly (TOSA) or, to be more precise, changing the temperature of the laser cavity. There is a temperature control circuitry inside the SFP, which is controlled by a microcontroller. The actual change of temperature is done via a TEC controller; which is connected to the microcontroller where it gets its set-point from. The actual wavelength tuning performed by the user is done by changing the tuning value in the microcontroller memory, which is translated to the temperature setting of the TEC and thus the TOSA temperature. All the communication is done via the I2C bus of the SFP transceiver. As observed at Nikhef by means of a wavelength meter (model AQ6140) measuring the behaviour of various OESolutions SFPs, the tuning mechanism permits to shift the wavelength of approximately $100 \mathrm{pm} /{ }^{\circ} \mathrm{C}$ as expected. After a warm up time of the SFP of 15 minutes, the laser temperature was changed in order to observe a wavelength shift of $\pm 100 \mathrm{pm}$. We recorded several DAC factory settings before and after the wavelength tuning, verifying the precise control mechanism. The results show a maximum wavelength variation of $6 \%$ between different SFP transceivers whose wavelength was shifted by $-100 \mathrm{pm}$ and a $10 \%$ maximum variation for $+100 \mathrm{pm}$ wavelength shift of the same SFP under test. At Nikhef several checks of the SFP alarm controls were performed, the SFP transceivers have warning/alarm controls which shuts down the laser emission if the laser temperature drifts more than $1{ }^{\circ} \mathrm{C}$ from the setpoint. As shown in Fig. 2 the measurements were focused on the SFP wavelength response checking the biggest tuning step without raising any alarm. The results show a fine control of these devices without raising alarms.

\section{Device aging and wavelength drift}

Long term device reliability is characterized by the increase in threshold current and the decrease in optical power [6]. Aging of the laser is associated with loss of optical efficiency resulting in a drop of optical power and a shift in the transmitted wavelength whereas it is preferable to operate the device in 


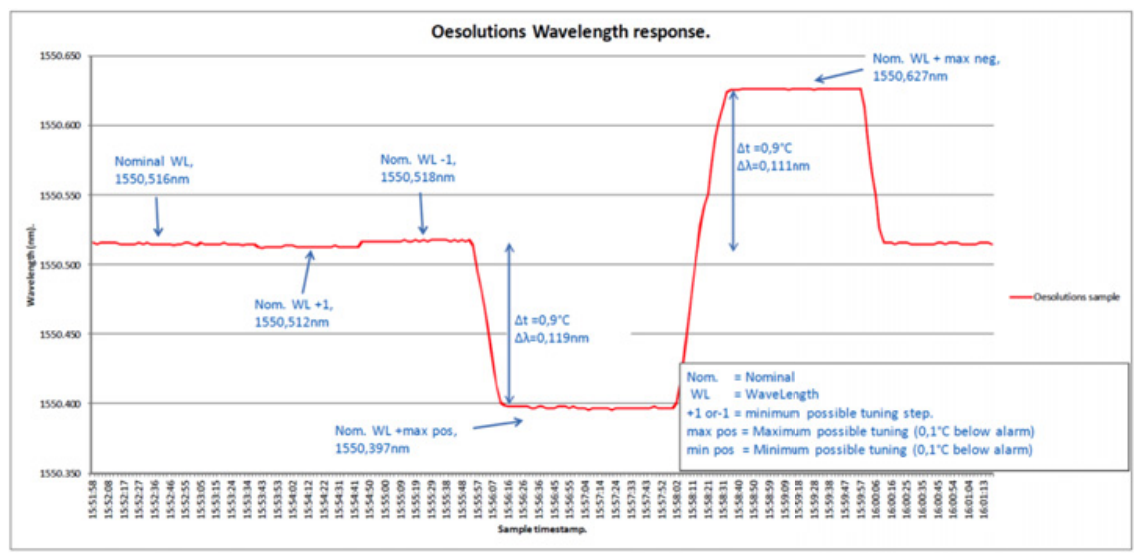

Figure 2. An example of SFP wavelength response changing the laser temperature (which time samples are reported on the $\mathrm{x}$-axis).

constant output power mode. As the device undergoes aging the dissipated power increases resulting in a rise at the junction temperature to which the lasing wavelength is sensitive. The lasing wavelength of a Distributed FeedBack (DFB) laser diode is given by the simple equation:

$$
\lambda=2 n \Lambda
$$

where $n$ is the effective refractive index and $\Lambda$ the grating pitch. In order to obtain accurately the desired wavelength, both parameters have to be well controlled. Moreover, the effective refractive index can change with driving conditions such as laser temperature or laser current. Due to the aforementioned reasons, temperature control is often used to fine-tune the DFB wavelength precisely to an ITU-T grid [7]. Over the device lifetime, the laser diode will exhibit wavelength drift. Eventually the wavelength drift could drive the channel into the neighbouring ones and then disrupting the communication. For these reasons we have studied various SFP transceiver devices in order to "retune" the possible wavelength drift in the future.

\section{Preliminary results}

The measurements performed at LNS (Laboratori Nazionali del Sud) aimed at studying the behaviour of different SFP transceivers varying the laser temperature by means of the Floor Control Module (FCM). This board is the tower floor control module: it permits to collect digital streams of data from 6 PMTs and 2 hydrophones located at the tower floor and collect data from local sensors (Temperature, Relative Humidity, Currents and Voltage, etc.) installed on the same board. The test setup is composed of a SFP transceiver plugged in the FCM and whose optical output signal goes to an Optical Spectrum Analyzer (OSA) for monitoring. Dedicated software has been written in the FCM firmware in order to read and write the SFP registers. A host PC is connected through RS232 to the FCM board with the OEsolutions tuning procedure implemented for tuning. The tuning procedure first foresees a warm up time of 15 minutes in order to stabilize the laser temperature. After checking the DAC settings and storing the factory tuning value, we disable the transceiver alarms and start a temperature scan. There will be four acquisitions at the nominal wavelength at the beginning and at the end of the tuning procedure (in order to check the wavelength stability before and after the scan). The temperature will be changed for 8 samples following a slant trend of $2{ }^{\circ} \mathrm{C}$ steps (each temperature step corresponds to half a channel according to the ITU-T grid). The tuning scan starts from the lower point and reach the 

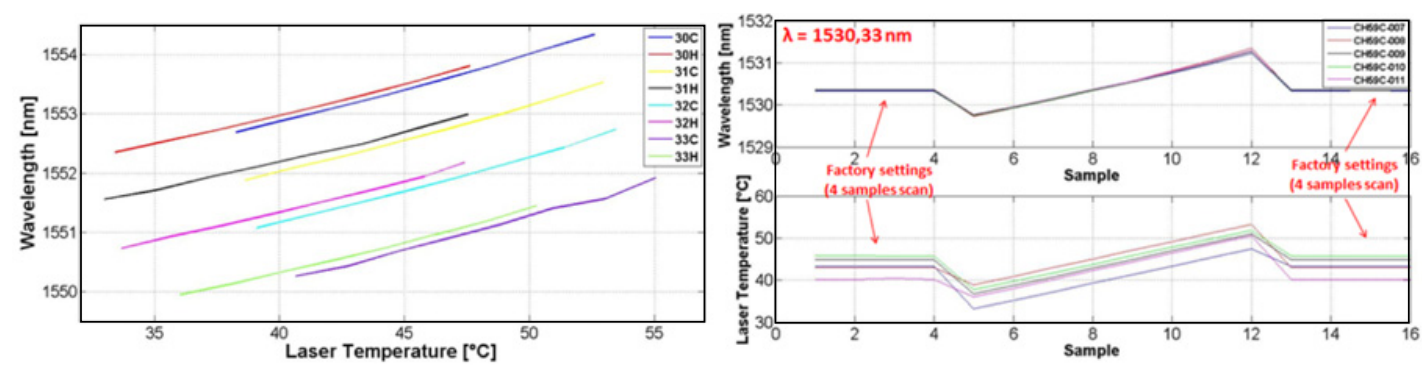

Figure 3. Left: various SFP transceiver trends of Wavelength $[\mathrm{nm}]$ vs. Laser Temperature $\left[{ }^{\circ} \mathrm{C}\right]$. Right: Five transceivers $\mathrm{CH} 59 \mathrm{C}$ measured, on top the wavelength measured values and on the bottom the laser temperature.

upper point in about three minutes. Figure 3 shows on the left an example of 8 samples tuning of eight transceivers from channel $30 \mathrm{C}$ to channel $33 \mathrm{H}$. Changing the tuning value changes the laser temperature and consequently the wavelength. In the right plots five $\mathrm{CH} 59 \mathrm{C}$ transceivers were taken, i.e. devices with the same nominal frequency. It can be seen that when the wavelength ramps are kept the same (top figure on the right) the temperature trends are not the same (bottom figure on the right). The preliminary results show that the relation between wavelength and temperature is not linear. The laser cavity non linearity could affect this behaviour, so it seems not easy to find a common behaviour between different devices under test.

\section{Conclusions}

The KM3NeT telescope is under construction and tests on the optical communication link, based on SFP transceivers, are still on going. Next step will be to monitor the transceivers on a long period and check the wavelength stability at high temperature in order to predict their behaviour. Characterizing more devices in the whole $\mathrm{C}$ band within $\pm 100 \mathrm{pm}$ would allow us to apply a wavelength correction if more than \pm 50 pm drift occur.

\section{References}

[1] T. Chiarusi, S. Aiello, F. Ameli, M. Anghinolfi, G. Barbarino, E. Barbarito, F. Barbato, N. Beverini, S. Biagi, B. Bouhadef et al., Journal of Instrumentation 9, C03045 (2014)

[2] A. Margiotta, Journal of Instrumentation 9, C04020 (2014)

[3] https://www.itu.int/rec/T-REC-G.694.1/en

[4] https://http://www.km3net.org/TDR/TDRKM3NeT.pdf

[5] http://www.tallgrass.nl

[6] J.S. Huang, Applied Physics Research 4, p15 (2012)

[7] M. Funabashi, H. Nasu, T. Mukaihara, T. Kimoto, T. Shinagawa, T. Kise, K. Takaki, T. Takagi, M. Oike, T. Nomura et al., Selected Topics in Quantum Electronics, IEEE Journal of 10, 312 (2004) 